This is the author's version of the work. It is posted here by permission of the AAAS for personal use, not for redistribution. The definitive version was published in Science on 12 March 2021, Vol 371, Issue 6534, with DOI: 10.1126/science.abg6514.

The personal author copy is available in final published form through a direct link provided by AAAS to be deposited at our institutional website and exclusively linked from it: https://www.meteoceanics.pt/wp/publications/science-abg6514/

\title{
Who is stirring the waters?
}

\section{Emerging pathways could improve attribution of changes in river flow across the globe}

\author{
Julia Hall ${ }^{1}$ and Rui A. P. Perdigão ${ }^{1,2,3}$ \\ 1 Meteoceanics Institute for Complex System Science, Vienna, Austria \\ 2 cE3c, Faculdade de Ciências,Universidade de Lisboa, Lisbon, Portugal. \\ 3 Instituto de Telecomunicações, Instituto Superior Técnico, Universidade de Lisboa, Lisbon, Portugal. \\ Email: hall@meteoceanics.pt; perdigao@meteoceanics.pt \\ Both authors contributed equally to this work.
}

Climate change, water, and land management affect the terrestrial water cycle and river flow. They do so through changes in precipitation and evaporation, aside from a multitude of other land surface processes. Earth system models are routinely used to simulate and detect globally observed changes and attribute these changes to climate change. Attribution is based on an assessment of the consistency or inconsistency of change signatures by including or excluding hypothesized drivers of change in processbased models (1). On page 1159 of this issue, Gudmundsson et al. (2) compare the consistency that globally observed trend-patterns in mean river flow and hydrological extremes exhibit with regard to a set of model simulations.

Gudmundsson et al. conclude, on the basis of the ESM output, that the simulated effects of water and land management cannot reproduce the observed change pattern in river flow. Rather, the modeled changes in river flow are only consistent with the observed changes in climatic variables if historical radiative forcing that accounts for climate change is used.

This finding is distinct and important, although Gudmundsson et al.'s attribution of changing river flow patterns to anthropogenic climate change is made by a simple 
quantitative line of arguments. For instance, if the model is driven by observational atmospheric forcing and it reproduces the observed global change pattern, the authors concluded that the observed trends are related to changes in the radiative forcing. If the observed changes are only consistent with model output driven with historical atmospheric forcing, then these trends are attributed to that driver.

Although the attribution statement in Gudmundsson et al. is logical and likely in terms of process understanding of climate dynamics, technically that evidence is still circumstantial. Indeed, different causal pathways could still lead to a similar outcome, that is, the same trend observed in the data could have emerged from a different process, even though not accounted for in the models. Additionally, owing to the presence of internal variability, such attribution will always have some degree of uncertainty (even with complete consistency between models and data) (1).

To improve the explanatory power of such important studies and to generate more confidence in such attribution statements, we need to move beyond these first-order assessments that involve simple proof of consistency and inconsistency when investigating the effects of climatic change.

The key for a more robust way to elicit the most likely driving mechanisms resides in characterizing the information transfer between potential drivers and the process of interest (e.g., between climatic change and river flows). Those providing strongest information transfer can be attributed as dominant drivers. Additionally, these information transfer metrics are probabilistic, hence internal variability and uncertainties are natively incorporated. This strengthens the process of attribution and makes it more realistic and reliable.

To achieve robust attribution, several measures of information transfer are already used elsewhere, including transfer entropy (3), traditional Bayesian approaches (4), and network connectivity metrics with time directionality $(5,6)$.

Attribution procedures by information transfer and Bayesian approaches are traditionally perceived as indicators of causality. However, they only allow quantifying the ability to infer the state of a process given the knowledge of another. Whether or not there is a cause-effect relation remains elusive, because no physical causation mechanism can be retrieved from these inferential statistics alone.

More recently, dynamical system metrics have been proposed with the aim to assess causal codependencies between drivers and processes (7) by evaluating whether there is a deterministic link between them (connection in phase space). This brings the added value of dynamic connectivity and allows for seamless integration with modeling approaches. However, even with these more advanced measures, a true cause-effect diagnostic is still elusive because the phase spatial diagnostics are basically correlative. The connected variables can simply be dynamically correlated effects of a common third-party cause.

The way forward is therefore to combine information transfer and dynamical system approaches, with fundamental principles and methodological understanding in mind. 
Such a combined approach allows bridging the best of both worlds while overcoming the respective caveats.

This brings us to the emerging pathways of information physics (8), reconciling and generalizing statistical, geometric, and mechanistic information metrics (9). The use of information physics enables the retrieval of physically consistent information attributes and dependencies in coevolutionary systems such as in hydrology and Earth system dynamics in a changing climate. Information physics can pave the way for bringing physical meaning to inferential metrics, and a dynamic coevolving flexibility to the statistical metrics of information transfer, bringing new pathways for causal discovery and attribution.

Exploring such pathways may thus provide further validation to the findings presented by Gudmundsson et al. and might also bring out unknown unknowns to add to the discussion of drivers of change in the hydrological system. This may thus complement any measure of causality that entails the development of multiple working hypotheses based on a thorough process-based understanding to avoid overlooking potential drivers of change that might cause the same signature (10).

The findings by Gudmundsson et al. allow one to infer that climate change has affected low, mean, and high flows at the global scale. Whether the retrieved drivers are the real causes or just predictors requires further investigation, and the development and application of causal discovery methods grounded on information physics offer encouraging pathways to further that quest for attribution.

\section{References}

1. N. L. Bindoff et al., in Climate Change 2013: The Physical Science Basis. Working Group I Contribution to the Fifth Assessment Report of the Intergovernmental Panel on Climate Change, T. F. Stocker et al., Eds. (Cambridge Univ. Press, 2013), p. 872.

2. L. Gudmundsson et al., Science 371, 1159 (2021).

3. T. Schreiber, Phys. Rev. Lett. 85, 461 (2000).

4. N. Najibi, et al., NPJ Clim. Atmos. Sci 2, 19 (2019).

5. J. Runge et al., Nat.Commun. 6, 8502 (2015).

6. A. E. Goodwell et al., Proc. Natl. Acad. Sci. U.S.A. 115, E8604 (2018).

7. S. Vannitsem, P. Ekelmans, Earth Syst. Dynam. 9, 1063 (2018).

8. R. A. P. Perdigão et al., Water Resour. Res. 56, e2019WR025270 (2020).

9. R. A. P. Perdigão, Entropy 20, 26 (2018).

10. S. Harrigan, C. Murphy, J. Hall, R. L.Wilby, J. Sweeney, Hydrol. Earth Syst. Sci. 18, 1935

(2014).

\section{Acknowledgements}

We acknowledge the Meteoceanics research programs MR-220617 "Mathematical Physics and Predictability of Complex Coevolutionary Systems" and MR-010319 "Synergistic Dynamics of Complex Socio-Natural Systems." R.A.P.P. also acknowledges the "Fundação para a Ciência e Tecnologia" through projects UIDB/00329/2020, UIDP/00329/2020, and UID/EEA/50008/2019. Both authors contributed equally to this work. 